\title{
Limitations of CT During PET/CT
}

\author{
Marc J. Gollub ${ }^{1}$, Richard Hong ${ }^{2}$, Debra M. Sarasohn ${ }^{1}$, and Tim Akhurst ${ }^{3}$ \\ ${ }^{1}$ Department of Radiology, Memorial Sloan-Kettering Cancer Center, New York, New York; ${ }^{2}$ Department of Radiology, \\ New York-Presbyterian Hospital/Weill Cornell Medical Center, New York, New York; and ${ }^{3}$ Department of Nuclear Medicine, \\ Memorial Sloan-Kettering Cancer Center, New York, New York
}

Our aim was to determine the diagnostic limitations of low-dose, unenhanced CT scans performed for anatomic reference and attenuation correction during PET/CT. Methods: The Radiology Information System at our oncologic hospital was queried during the 9-mo period from July 2002 to April 2003 for patients with PET/CT scans and diagnostic enhanced CT within 2 wk of each other. One radiologist interpreted the CT portion of the $\mathrm{PET} / \mathrm{CT}\left(\mathrm{CTP}^{\mathrm{P}}\right)$ unaware of the PET results and the associated enhanced diagnostic $C T\left(C T^{d}\right)$. A medical student compared this interpretation with the official report of the $\mathrm{CT}^{\mathrm{d}}$ and listed all discrepancies between reports. A separate radiologist compared $\mathrm{CT}^{\mathrm{p}}$ and $\mathrm{CT}^{\mathrm{d}}$ images and classified true discrepant findings as due to lack of intravenous contrast, arm-position artifact, lack of enteric contrast, low milliamperage $(\mathrm{mA})$, and quality of lung images. Results: Among 100 patients, the most common malignancies were lymphoma $(n=37)$, cancer of the colorectum ( $n=$ 31 ), and esophageal cancer $(n=15)$. Among 194 true discrepancies in which findings were missed at CTP, causes were as follows: (a) lack of intravenous contrast (128/194, 66\%), (b) armdown artifact $(17 / 194,9 \%)$, (c) quality of lung images (26/194, $13 \%)$, (d) lack of enteric contrast (15/194, 8\%), and (e) low mA $(8 / 194,4 \%)$. Discrepancies were seen most commonly in detecting lymphadenopathy and visceral metastases. Conclusion: Most missed findings on the unenhanced CT portion of the PET/CT scans were due to technical factors that could be altered. Discrepant findings would have led to altered management in only 2 patients, suggesting a role for limited repeat imaging to reduce radiation and use of valuable resources.

Key Words: PET/CT; diagnostic CT; limitations of CT

J Nucl Med 2007; 48:1583-1591

DOI: 10.2967/jnumed.107.043109

D

iagnostic CT scanning has been the mainstay of oncologic imaging over the past $25 \mathrm{y}$. By the end of the 1990s, ${ }^{18}$ F-FDG PET/CT was developed, offering the first opportunity to combine metabolic and anatomic information leading to a powerful new combined-modality imaging examination.

Methodologic and technical differences between the CT performed for anatomic localization and attenuation correc-

Received Apr. 27, 2007; revision accepted Jul. 2, 2007

For correspondence contact: Marc J. Gollub, MD, Room C 276F, Department of Radiology, Memorial Sloan-Kettering Cancer Center, 1275 York Ave., New York, NY 10021.

E-mail: gollubm@mskcc.org

COPYRIGHT @ 2007 by the Society of Nuclear Medicine, Inc. tion $\left(\mathrm{CT}^{\mathrm{p}}\right)$ and a typical diagnostic $\mathrm{CT}$ scan $\left(\mathrm{CT}^{\mathrm{d}}\right)$ include arm position (frequently down for $\mathrm{CT}^{\mathrm{p}}$ ), suspended respiration (vs. quiet breathing for $\mathrm{CT}^{\mathrm{p}}$ ), use of enteric contrast (variable in $\mathrm{CT}^{\mathrm{p}}$ ) and intravenous contrast materials (not typically used in $\mathrm{CT}^{\mathrm{p}}$ ), and levels of microamperage-seconds $(\mathrm{mA} \cdot \mathrm{s})$ of radiation (much lower in $\mathrm{CT}^{\mathrm{p}}$ ).

The limitations of the $\mathrm{CT}^{\mathrm{p}}$ are partly intuitive, based on the increased accuracy of CT using oral and intravenous contrast agents noted early on in the development of CT protocols (1). The functional information provided by PET when correlated with the CT anatomic information, in some clinical scenarios, served to overcome expected limitations incurred with the absence of intravenous or oral contrast. This has been most obvious in patients with lymphoma, where nodal disease status is not well predicted by size or morphology on CT as much as by metabolic activity on ${ }^{18} \mathrm{~F}$-FDG PET; thus, diagnostic CT scans have added little useful information beyond the anatomic coregistration provided (2). This has led to a proposed change in diagnostic approach, at least in lymphoma, wherein ${ }^{18}$ F-FDG PET might be thought of as the only imaging test required. Aside from these direct investigations in patients with lymphoma, and the theoretic limitations of these low-dose, localizing, unenhanced CT scans, little practical data are available with regard to the limitations of this $\mathrm{CT}^{\mathrm{p}}$ and the advantage of diagnostic $\mathrm{CT}$ in addition to PET/CT for other types of malignancies. Might a similar shift in diagnostic strategy with limited additional scanning be possible in patients with other types of malignancies?

The goal of this investigation was to document limitations of $\mathrm{CT}^{\mathrm{p}}$ more specifically and to assist imaging specialists who may be less familiar with these limitations. By documenting the limitations in various organ systems and for various tumor types, we aimed to outline the advantages of either PET/CT with intravenous contrast or, if appropriate, suggest the need for limited additional diagnostic contrast-enhanced CT. Specifically, we aimed to elucidate what findings could be missed if only $\mathrm{CT}^{\mathrm{p}}$ were performed in patients with various malignancies and how, if at all, this might affect their clinical course.

\section{MATERIALS AND METHODS}

\section{Patients}

This was a retrospective study approved by our institutional review board. Patient consent was deemed unnecessary. 
We queried our Radiology Information System (Quadris, version 981218; ADAC HealthCare Information Systems) over a 9-mo period (July 2002 to April 2003) to find PET/CT scans performed consecutively at our institution. Inclusion criteria were patients with cancer who also had a diagnostic CT scan of the abdomen with or without the pelvis or chest with intravenous and oral contrast material also performed at our institution in patients of any age within 2 wk before or after the PET/CT.

\section{PET/CT}

All patients fasted for at least $6 \mathrm{~h}$ before imaging. All images were obtained with a dedicated PET/CT system that operates in 2-dimensional mode, has an axial field of view of $15.5 \mathrm{~cm}$, and has an axial slice thickness (resolution) of 4.2-mm (full width at half maximum) intensity at the center of the field of view (Discovery LS; GE Healthcare). Images were acquired after intravenous injection of $555 \mathrm{MBq}(15 \mathrm{mCi}){ }^{18} \mathrm{~F}-\mathrm{FDG}$ and a $45-$ to 60 -min uptake period and were reconstructed with a 28-subset, 2-iterations algorithm, $128 \times 128$ matrix, and CT-based attenuation coefficient. For anatomic localization and attenuation correction, CT (CTp) was performed without intravenous iodinated contrast. Oral contrast, diatrizoate meglumine/sodium ([MD-Gastroview; Mallinckrodt] in $1,000 \mathrm{~mL}$ sterile water with raspberry ice Crystal Light, $2.26 \mathrm{~g}$ [Kraft Foods, USA]) or 2.1\% (w/v) barium sulfate suspension, $2.0 \%$ (w/w) (Readi-Cat 2; EZ EM), was administered to 13 patients, 45 min before scanning. Scanning parameters included $120-140 \mathrm{kVp}$ (kilovoltage peak), 64 average $\mathrm{mA} \cdot \mathrm{s}, 5-\mathrm{mm}$ slice thickness, and 4.25-mm table increment. Patients were scanned supine with their arms at their sides during quiet respiration.

\section{Diagnostic CT (CT $)$}

Within 2 wk before or after PET/CT, CT ${ }^{\mathrm{d}}$ was performed on a multislice helical scanner with either 4- or 8-detector rows using $140 \mathrm{kVp}$, variable $\mathrm{mA} \cdot \mathrm{s}$ depending on body habitus (average $=$ $290 \mathrm{~mA} \cdot \mathrm{s}$ ), beam pitch $0.9375-1.375,0.5$ - to 0.8 -s tube rotation speed, and 5-mm slices (Lightspeed and Lightspeed Ultra; GE Healthcare). Oral contrast, 1,000 mL, was administered 45-60 min before scanning (diatrizoate meglumine and diatrizoate sodium solution [Gastroview]; Mallinckrodt). In patients who received oral contrast immediately before for $\mathrm{CT}^{\mathrm{P}}, 8-16 \mathrm{oz}$ additional contrast was administered to fill the stomach. Nonionic intravenous contrast, $150 \mathrm{~mL}$, was administered at a rate between
1.5 and $4.0 \mathrm{~mL} / \mathrm{min}$, depending on venous access and scan type (Omnipaque 300; GE Healthcare).

\section{Image Analysis}

One body imager with $>10$-y experience in interpreting body CT interpreted the $\mathrm{CT}^{\mathrm{P}}$ unaware of the images and report of the associated PET scan and the comparison $\mathrm{CT}^{\mathrm{d}}$ performed within 2 wk. Results were directly transcribed by a medical student. All studies were visualized on a PACS (Centricity PACS 2.1; GE Healthcare), using the scroll function and with liberal use of variable window/level settings to overcome the limitations of the lack of intravenous contrast.

Subsequently, the medical student compared the transcribed official report of the $\mathrm{CT}^{\mathrm{d}}$ with the interpretation of the matching $\mathrm{CT}^{\mathrm{p}}$ and listed discrepant findings by anatomic region and organ system, noting the finding, date of the scan, and image number. Patient demographics and primary malignancy were also recorded.

A second abdominal imager with $>5$ y experience interpreting abdominal CT was prompted to classify each discrepant finding by performing a side-by-side comparison of $\mathrm{CT}^{\mathrm{P}}$ and $\mathrm{CT}^{\mathrm{d}}$ on PACS. Discrepancies were identified as 5 "true" discrepancy categories: "IV", "Lung", "Arm-down", "Enteric", and "Low mA". Other discrepancies were identified as 4 "false" discrepancy categories: "Insignificant", "Perceptive", "Interpretive", and "Patient". "IV" was defined as a discrepancy that was thought to be due to the lack of intravenous contrast used in the $\mathrm{CT}^{\mathrm{p}}$ (e.g., nonenhancement of liver lesions, inability to distinguish lymph nodes from vessels or other structures) (Fig. 1). "Lung" was defined as a discrepancy due to either the lack of use of an edge-enhancing algorithm ("lung" kernel) to reconstruct the images (in which a nodule or focus of ground-glass opacity was unable to be resolved) or due to respiratory artifact (e.g., motion, poor inspiration, mosaic perfusion, etc.) typically seen at non-breathold CTp (Fig. 2). "Arm-down” was defined as a focal beam-hardening artifact obscuring findings on the $\mathrm{CT}^{\mathrm{p}}$ that were well seen on $\mathrm{CT}^{\mathrm{d}}$ (Fig. 3). "Enteric" was defined as a finding missed due to a lack of administration of oral or rectal contrast on the $\mathrm{CT}^{\mathrm{p}}$ (e.g., bowel, mesenteric, and nodal lesions) (Fig. 4). "Low mA" was defined as a finding not appreciated on the $\mathrm{CT}^{\mathrm{P}}$ because of overall graininess of the image (global beam-hardening) (Fig. 5). "Patient" was defined as a change in radiographic findings that could be explained by a change in the patient's clinical condition in the time interval (0-14 d) between $\mathrm{CT}^{\mathrm{P}}$ and $\mathrm{CT}^{\mathrm{d}}$ (e.g., development of
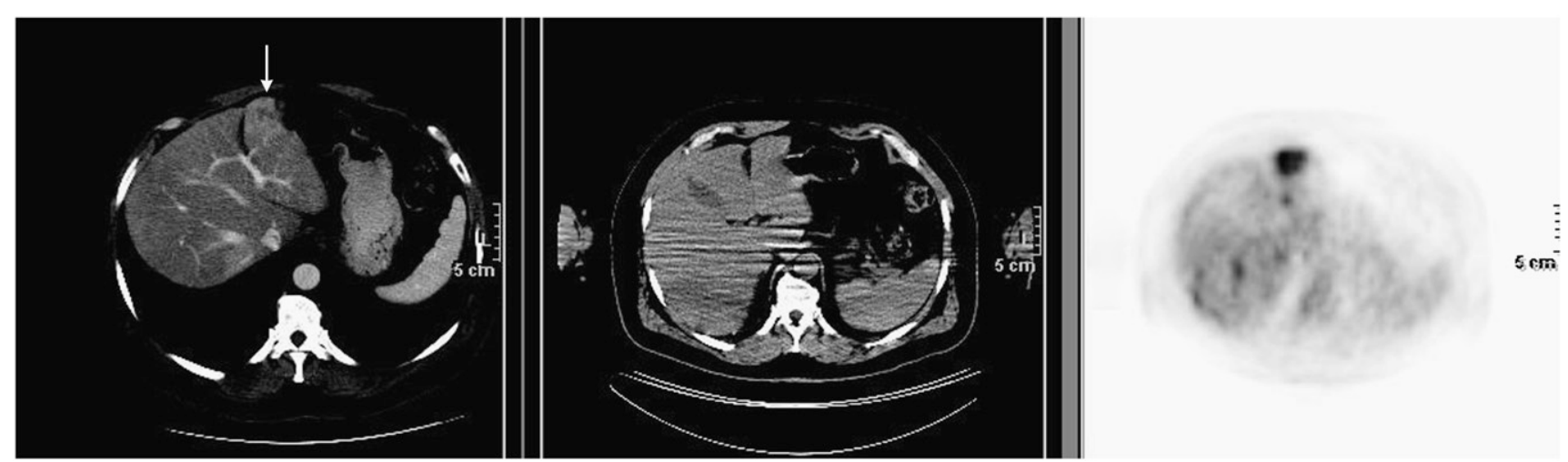

FIGURE 1. Patient with colorectal cancer with hepatic metastasis in left hepatic lobe seen on CT ${ }^{d}$ (arrow) but not on $\mathrm{CT}^{\mathrm{P}}$. Corresponding PET image confirms metastasis. This was considered a potentially significant finding. Treatment was not changed as it was seen on PET portion of scan. 


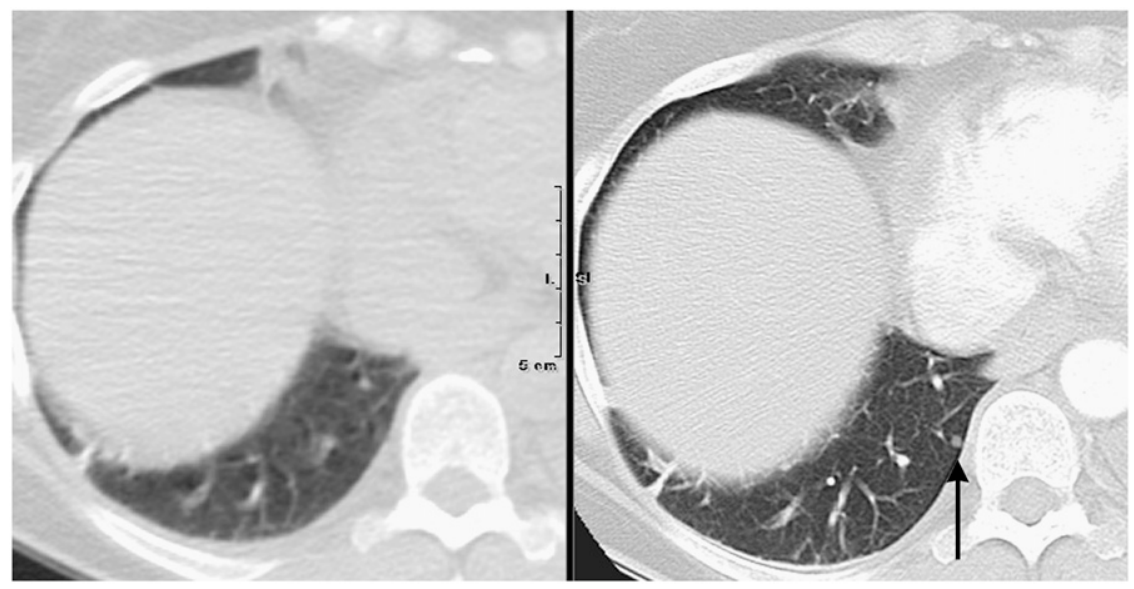

FIGURE 2. Patient with non-small cell lung cancer and increasing metastatic disease with new lung and liver (not shown) lesions. Small subpleural nodule (arrow) was not seen on CTP likely due to both breathing and non-edge-enhancing algorithm. Note that such a nodule would be unlikely to be seen on PET because of its small size, and its visualization on PET/CT would rely on better-quality lung images only. This was not considered a significant finding as generalized increase in metastatic disease was noted.

pleural effusions or lung infiltrates or ascites). "Insignificant" was defined as a clinically unimportant incidental finding (e.g., bone island or a subcentimeter renal low-density lesion too-small-to characterize mentioned in only 1 report, either $\mathrm{CT}^{\mathrm{d}}$ or $\mathrm{CT}^{\mathrm{p}}$, etc.). "Perceptive" difference was defined as an abnormality that was present on both $\mathrm{CT}^{\mathrm{p}}$ and $\mathrm{CT}^{\mathrm{d}}$ but was not mentioned in the review of CT ${ }^{p}$ because of oversight. "Interpretive difference" was defined as an abnormality that was present on $\mathrm{CT}^{\mathrm{p}}$ and $\mathrm{CT}^{\mathrm{d}}$ but was of a more subjective nature and interpreted differently (e.g., prostate enlargement, cardiomegaly, splenomegaly, or adrenal thickening).

Further analysis consisted of review of the official PET scan report, PET images as needed, and the electronic medical record for clinical notes, laboratory values, surgical and biopsy results, and follow-up imaging results for 36 mo.

On the basis of this chart review of all true discrepancies, the following were determined by one attending physician and the radiology resident (medical student at beginning of project): (i) which discrepant findings were of potential clinical significance (e.g., could change the cancer stage or treatment [based on the accepted approach for staging and treatment at the authors' institution] because of increase or decrease in existing disease or new disease appearing or could require intervention because of infection, hemorrhage, or bowel, biliary, or renal obstruction or deep venous thrombosis, aneurysm, fracture, etc.); (ii) which potentially significant discrepant findings, determined by $\mathrm{CT}^{\mathrm{d}}$ to have been overlooked on $\mathrm{CT}^{\mathrm{p}}$, were diagnosed nonetheless on the basis of PET findings; (iii) which potentially significant findings were missed both on $\mathrm{CT}^{\mathrm{p}}$ and PET but seen on $\mathrm{CT}^{\mathrm{d}}$; (iv) which patients, among group iii, incurred a change in management. A change in management was defined as a change in type, dose, or schedule of administration of chemotherapy or radiotherapy, cessation or commencement of the same plan or a new plan for surgery, excisional biopsy, or a change in extent or approach of surgery, or cancellation of surgery. Change in management could also include performance of an invasive procedure, commencement or cessation of anticoagulation, antibiotics, and other nononcologic medical or surgical treatment.

\section{RESULTS}

PET/CT and diagnostic CT scans of 100 patients (62 male, 38 female; average age, $55.6 \mathrm{y}$; range, 4-83 y) were reviewed. All PET/CT scans were full-body scans. Diagnostic CT scans encompassed the chest, abdomen, and pelvis $(n=85)$; the chest and abdomen only $(n=10)$; and the abdomen and pelvis only $(n=5)$. Malignancies included the following: lymphoma $(n=37)$; colorectal cancer $(n=31)$; esophageal cancer $(n=15)$; 2 each of cholangiocarcinoma, lung cancer, neuroblastoma, and softtissue sarcoma; and 1 each of breast, gallbladder, gastric, hepatocellular, pancreatic, prostate, unknown primary cancers, and myelodysplastic syndrome and melanoma.

Thirteen patients received oral contrast on $\mathrm{CT}^{\mathrm{p}}(13 \%)$. Twenty-eight patients underwent $\mathrm{CT}^{\mathrm{d}}$ after PET/CT $(28 \%$; average, $5.3 \mathrm{~d}), 22$ underwent $\mathrm{CT}^{\mathrm{d}}$ before PET/CT $(22 \%$; average, $2.6 \mathrm{~d}$ ), and 50 underwent $\mathrm{CT}^{\mathrm{d}}$ on the same day.

There were 482 total discrepancies between the interpretation of the $\mathrm{CT}^{\mathrm{p}}$ and the dictated report of the $\mathrm{CT}^{\mathrm{d}}$. The average number of discrepancies per scan was $=4.8$ (range, 0-17). There were 288 discrepancies that did not

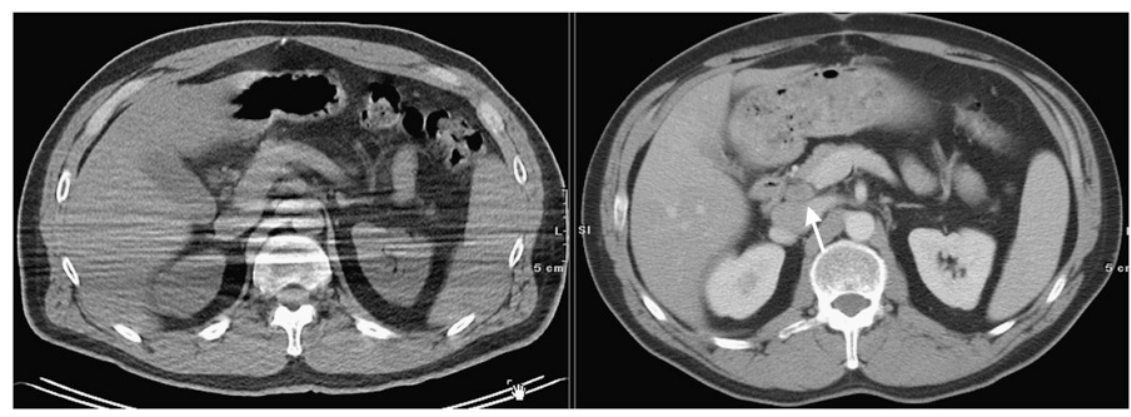

FIGURE 3. Patient with colorectal cancer with portacaval lymphadenopathy (arrow) obscured by localized beamhardening artifact from arm-down position. This was not considered a significant finding as it was unchanged and was seen on PET. 
FIGURE 4. Patient with lymphoma and nodules in terminal ileum (arrow). These are not appreciated on $\mathrm{CT}^{\mathrm{P}}$ without benefit of oral contrast. This finding was considered clinically significant as it could represent extranodal spread of lymphoma. It was proven to be Crohn disease after colonoscopy and surgical removal.

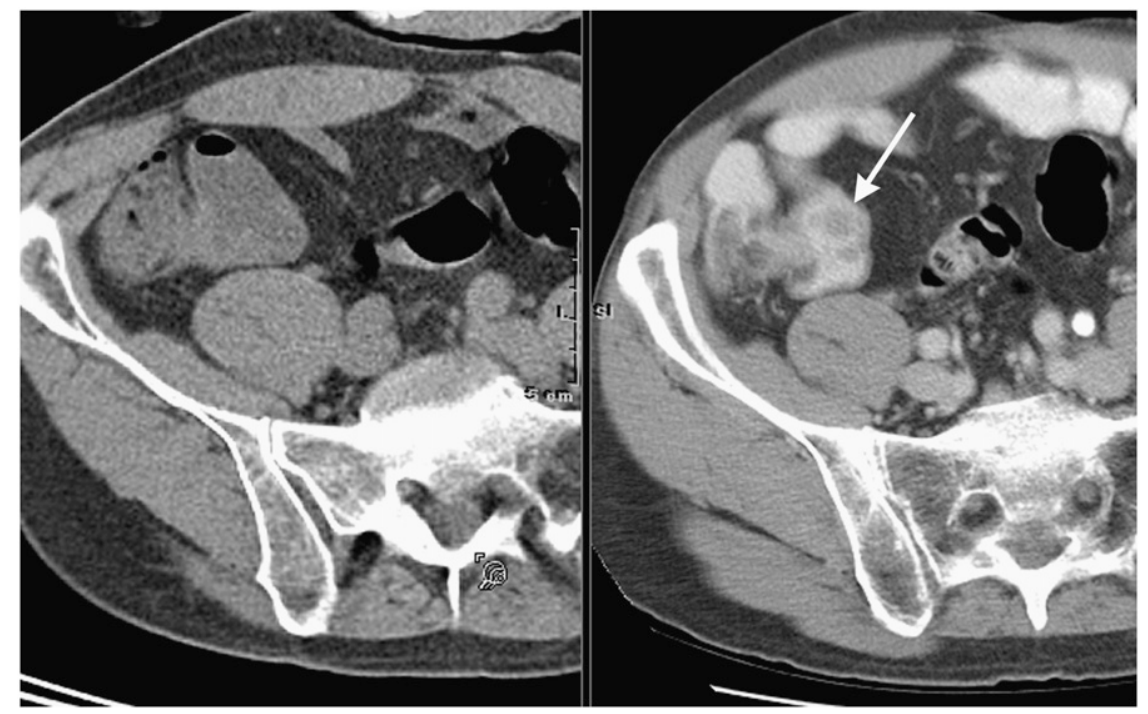

represent true discrepant findings between the $\mathrm{CT}^{\mathrm{p}}$ and the $\mathrm{CT}^{\mathrm{d}}$ scans, including 110 perceptive differences, 86 interpretive differences, 84 insignificant differences, and 8 changes in patients' clinical conditions. There were 194 true discrepancies (80 patients), with an average of 2.4 per patient (range, 1-8) (Table 1; Fig. 6). Sixty discrepancies (31\%) were judged to be potentially significant.

Twenty-six (43\%) of these 60 discrepant findings, though missed on $\mathrm{CT}^{\mathrm{p}}$, were diagnosed in 22 patients on the PET scan portion of the PET/CT in patients with colorectal cancer $(n=8)$, lymphoma $(n=7)$, esophageal cancer $(n=$ 3 ), and 1 each of cholangiocarcinoma, gallbladder cancer, neuroblastoma, and pancreatic carcinoma.

Thirty-four potentially significant findings missed on PET and on $\mathrm{CT}^{\mathrm{p}}$ but seen on $\mathrm{CT}^{\mathrm{d}}$ were noted in 24 patients with colorectal cancer $(n=10)$, lymphoma $(n=7)$, esophageal cancer $(n=3)$, and 1 each of cholangiocarcinoma, neuroblastoma, lung, and gastric cancer (Table 2). These occurred in lymph nodes $(n=8)$, viscera $(n=22)$, and soft tissues $(n=4)$. As can be seen in Table 2, the vast majority of these discrepancies were caused by the lack of intravenous contrast. This obscured the true size or number of hypo- and hypervascular liver metastases or of biliary dilatation or obscured the recognition of lymph nodes. Other reasons included focal beam-hardening from the arm-down position obscuring liver lesions or lymph nodes, obscuration of lung nodules from a smooth lung algorithm, and obscuration of bowel wall thickening or luminal masses from lack of oral contrast.

Chart review of these 34 cases indicated that most discrepant lesions were not pursued because of the widespread disease in these patients such that treatment would not have been altered. However, discrepant findings would have led to a change in management in 2 patients (2/46 patients, $4 \%$ ). The changes consisted of the performance of a cystoscopy in a patient with lymphoma to work up a mass missed on the CT and PET portions of the PET/CT near the bladder. The mass seen on $\mathrm{CT}^{\mathrm{d}}$ was worrisome for lymphoma versus bladder cancer (patient was a smoker). Cystoscopy was normal, and after chemotherapy (being given for lymphoma anyway) the mass shrunk, indicating that it was probably lymphoma. In a second patient with lymphoma, colonoscopy and surgical removal of the neoterminal ileum was required to diagnose the cause of the nodules (Crohn disease) in the ileum not seen on the CT or PET portions of the PET/CT due to the lack of oral contrast but well seen

FIGURE 5. Patient with esophageal carcinoma. Small regional lymph node (arrow) is suggestive of metastases because of location (not size). It is difficult to resolve from esophagus itself because of overall beam-hardening. This was not considered significant as liver and bone lesions were seen on PET.

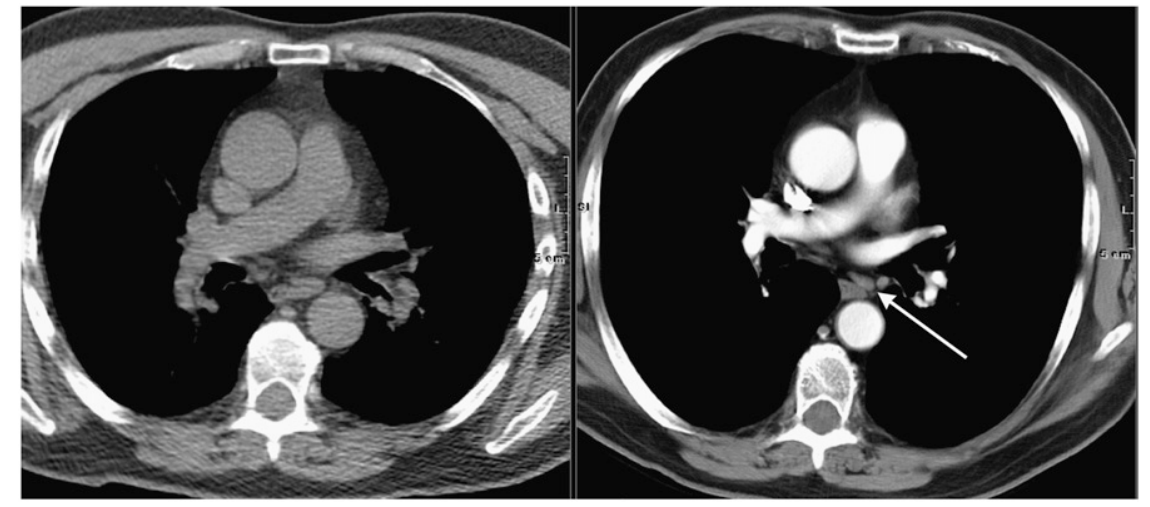


TABLE 1

Discrepancies Between CT ${ }^{p}$ and CT $^{d}(n=482)$

\begin{tabular}{llcc}
\hline Discrepancy & Category & $\begin{array}{c}\text { No. of } \\
\text { discrepancies }\end{array}$ & $\begin{array}{c}\text { Percentage } \\
\text { of true }\end{array}$ \\
True & discrepancies \\
\hline & IV & 194 & \\
& Lung & 128 & 66 \\
& Algorithm & 26 & 13 \\
& Respiratory & 14 & 7 \\
False & Arm-down & 12 & 6 \\
& Enteric & 15 & 9 \\
& Low mA & 8 & 8 \\
& Interpretive & 288 & 4 \\
& Insignificant & 84 & 30 \\
& Perceptive CTp & 78 & 29 \\
& Perceptive CT & & \\
& Patient change & 32 & 11 \\
& 8 & 3
\end{tabular}

on $\mathrm{CT}^{\mathrm{d}}$. Thus, the change in management of the 2 patients was due to use of invasive diagnostic examinations $(n=2)$ and the need for an operation $(n=1)$ (Fig. 7).

With respect to hepatic versus extrahepatic discrepancies for the 3 most common malignancies, colorectal cancer showed a fairly even distribution of overall true and potentially significant discrepancies between hepatic and extrahepatic sites; lymphoma and esophageal cancer showed a predominance of true and potentially significant discrepancies in extrahepatic over hepatic sites (Tables 3 and 4).

Pelvic region discrepancies were found in only 13 patients. Six were deemed potentially significant, of which 3 were noted on PET. One finding led to the need for an additional procedure (change in management) (Table 5).

\section{DISCUSSION}

The development of whole-body PET scanners using readily available tracers such as ${ }^{18}$ F-FDG represented a tremendous contribution to the diagnostic armamentarium of cancer imaging. However, the metabolic information provided by ${ }^{18} \mathrm{~F}$-FDG uptake alone did not provide adequate image resolution to afford accurate anatomic localization of diseased areas. This prompted the development of combined PET/CT scanners (3), packaging multislice helical scanners (e.g., 16- to 64-row detector scanners) with whole-body PET scanners), and allowing high-quality multiplanar reformatted isotropic data for fusion with PET. Although the CT portion of the scan was used mainly for anatomic coordination and for rapid attenuation correction, it became apparent that it might contain valuable information, especially if oral and or intravenous contrast were administered. Controversy with regard to the administration of oral or intravenous iodinated contrast material exists due to technical, economic, workflow, and radiation safety concerns. In our institution, if diagnostic CT is desired, it may be done on the same machine for patient convenience just after the completion of the PET/CT with a dynamic injection of intravenous contrast. Oral contrast is routinely given for the localizing CT to assist in the localization of abnormalities within or adjacent to bowel. However, several institutions have also begun to administer intravenous contrast material with the PET/CT for the localizing scan to allow the "localizing" CT to become

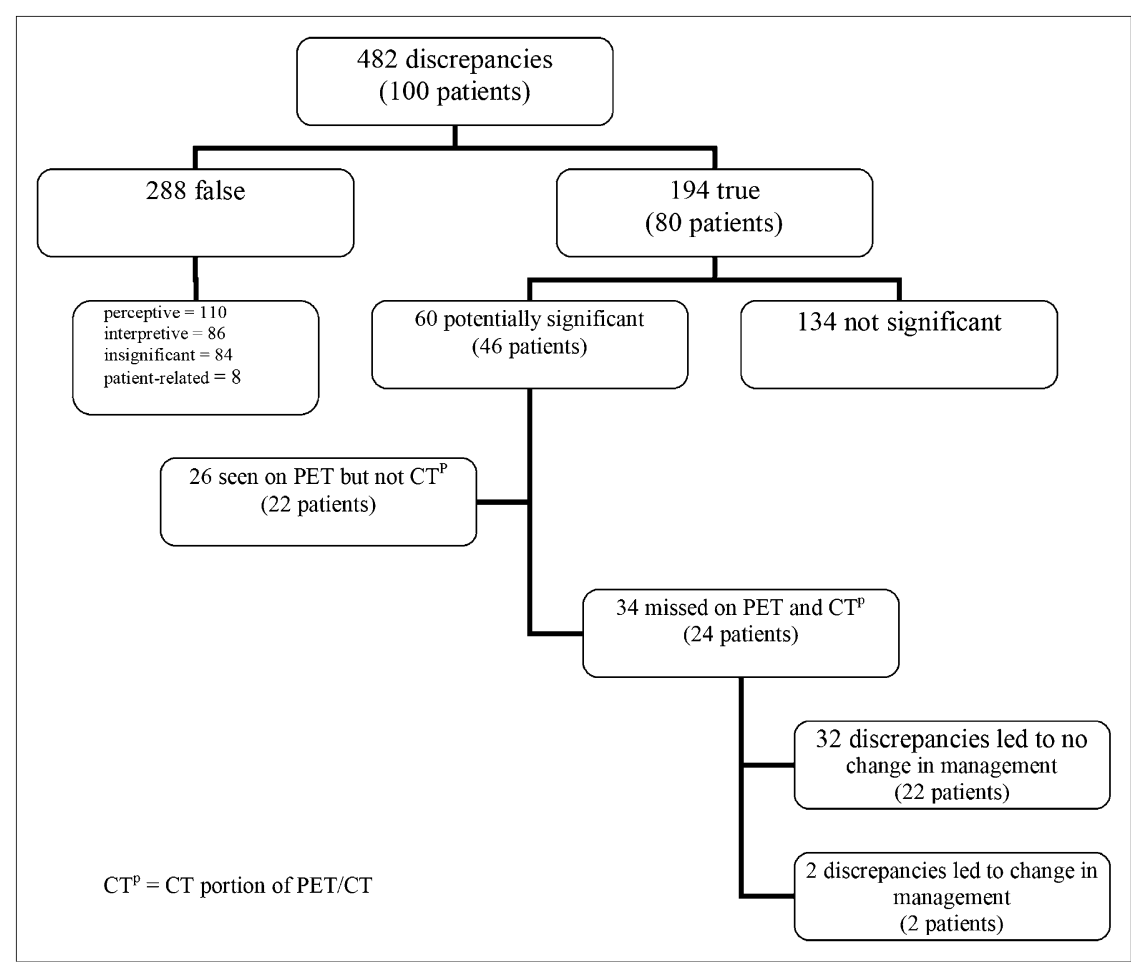

FIGURE 6. Box diagram outlining results of analysis of PET/CT scans by discrepancy and patient. 
TABLE 2

Potentially Significant Discrepant Findings Seen only on $\mathrm{CT}^{\mathrm{d}}(n=34)$

\begin{tabular}{|c|c|c|c|c|}
\hline Discrepant organ & No. & Malignancy & Description & Category \\
\hline Lymph nodes & 8 & & & \\
\hline \multirow[t]{3}{*}{ Chest } & 3 & Lymphoma & Paratracheal node, $0.7 \mathrm{~cm}$ & IV \\
\hline & & Neuroblastoma & Paratracheal node, $0.7 \mathrm{~cm}$ & IV \\
\hline & & Neuroblastoma & Bilateral axillary nodes, largest $1.3 \times 0.9 \mathrm{~cm}$ & Arm \\
\hline \multirow[t]{3}{*}{ Abdomen } & 3 & Colorectal & Periportal node, $2.8 \times 1.4 \mathrm{~cm}$ & IV \\
\hline & & Gastric & Perigastric node, $0.8 \mathrm{~cm}$ & IV \\
\hline & & Neuroblastoma & L paraaortic node, $0.6 \mathrm{~cm}$ & IV \\
\hline \multirow[t]{2}{*}{ Pelvis } & 2 & Neuroblastoma & $\begin{array}{l}\text { L common iliac, } 0.7 \times 0.5 \mathrm{~cm} / \mathrm{R} \text { external iliac nodes, } \\
0.6 \times 0.5 \mathrm{~cm}\end{array}$ & IV \\
\hline & & Colorectal & Sigmoid mesenteric nodes, $0.5 \mathrm{~cm}$ & $\mathrm{~mA}$ \\
\hline Viscera & 26 & & & \\
\hline \multirow[t]{2}{*}{ Lung } & 2 & Lung & Nodule, $0.6 \mathrm{~cm}$ & Lung algorithm \\
\hline & & Colorectal & Bilateral nodules, largest $0.7 \mathrm{~cm}$ & Lung algorithm \\
\hline \multirow[t]{16}{*}{ Liver } & 16 & Colorectal & Size discrepancy in hepatic metastases & IV \\
\hline & & Colorectal & Segment $4,2.5 \times 1.5 \mathrm{~cm}$ & IV \\
\hline & & Colorectal & Segment $5,1.7 \times 0.7 \mathrm{~cm}$ & Arm \\
\hline & & Colorectal & Segment $8,1 \times 0.6 \mathrm{~cm}$ & Arm \\
\hline & & Colorectal & New mild biliary dilatation & IV \\
\hline & & Lymphoma & Increased biliary dilatation & IV \\
\hline & & Esophageal & Segment $5,1.2 \times 1.2 \mathrm{~cm}$ & IV \\
\hline & & Colorectal & Segment $3,0.9 \times 0.3 \mathrm{~cm}$ & IV \\
\hline & & Colorectal & Increased severe $\mathrm{R}$ biliary dilatation & IV \\
\hline & & Cholangiocarcinoma & Segment $6 /$ hepatic dome, subcentimeter lesions & IV \\
\hline & & Lung & Segment $7,2.7 \times 2.3 \mathrm{~cm}$ & Arm \\
\hline & & Lung & Segment 2 , subcentimeter hypervascular foci & IV \\
\hline & & Colorectal & Scattered lesions, largest $2.8 \times 1.8 \mathrm{~cm}$ & IV \\
\hline & & Esophageal & Segment 2 , subcentimeter lesion & IV \\
\hline & & Esophageal & Segment $7 / 8,1.9 \times 1.1 \mathrm{~cm}$ hypervascular lesion & IV \\
\hline & & Esophageal & Segment 6 , subcentimeter hypervascular lesion & IV \\
\hline Kidney & 1 & Lymphoma & Slight delay in L kidney excretion & IV \\
\hline \multirow[t]{3}{*}{ Bowel } & 3 & Lymphoma & Soft tissue in gastric antrum & Oral \\
\hline & & Lymphoma & Filling defects in terminal ileum ${ }^{*}$ & Oral \\
\hline & & Esophageal & Gastric fundal thickening & Oral \\
\hline Soft tissue & 4 & & & \\
\hline Peritoneum & & Colorectal & Peritoneal carcinomatosis & Resp. Art. \\
\hline Chest & & Colorectal & Subcutaneous nodules & $\mathrm{mA}$ \\
\hline Chest & & Lymphoma & Postmediastinal soft tissue & IV \\
\hline Pelvis & & Lymphoma & Prevesical, $6.3 \times 3.3 \mathrm{~cm}$ soft-tissue mass ${ }^{*}$ & IV \\
\hline $\begin{array}{l}\text { *Management was } \\
\text { Resp. Art. = respi }\end{array}$ & arti & his case. & & \\
\hline
\end{tabular}

"diagnostic" and to avoid the necessity of a repeat examination with its additional radiation, cost, and use of valuable and potentially limited resources. In fact, attenuation correction tables have been created for contrastenhanced CT scans (4). This would seem to be the most economical protocol overall from the standpoint of time and radiation exposure, although there are reports that the intravenous contrast can result in an overestimation of the standardized uptake value (SUV) arising from elevated CT attenuation in areas of focal intravenous contrast accumulation, such as the vessels or parenchymal organs. The amount of the SUV overestimation is not significant in tumor (2,4-9). Several other investigators have recently confirmed the feasibility of using intravenous contrast in various types of modified injection protocols allowing combined diagnostic CT with PET (10-12).

Oral contrast may affect the CT-based attenuation $(2,13,14)$. The resulting attenuation-corrected PET activity can be overestimated by as much as $20 \%$, but with an average of $4.4 \%$ with standard dilutions of positive oral contrast $(13,15)$. Many centers either withhold oral contrast or administer a neutral density oral contrast to help distend and identify the intestines, without interfering with attenuation mapping (16).

Studies comparing staging information gained from PET/CT with diagnostic CT have shown that, for esophageal and colorectal cancer, impact on patient care from more accurate staging can occur in $20 \%-30 \%$ of cases 


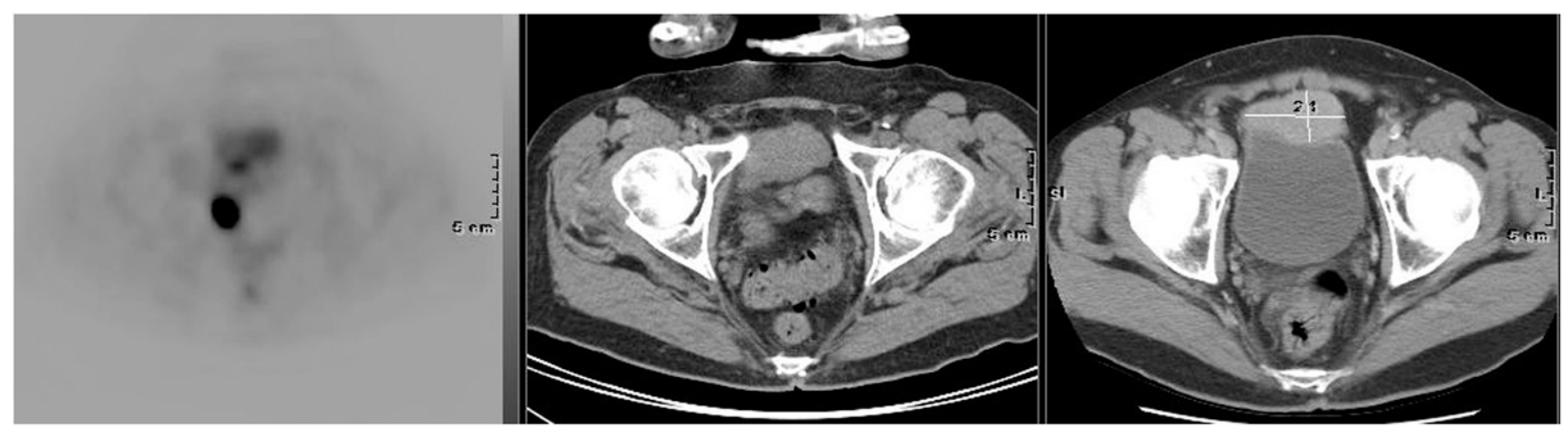

FIGURE 7. A 63-y-old man with lymphoma and mass above bladder with low standardized uptake value, not prospectively identified on PET or CTP but well seen on $\mathrm{CT}^{\mathrm{d}}$. Note lower bladder volume on PET/CT.

$(17,18)$. Some studies have even suggested that in tumors such as lymphoma and gastrointestinal stromal tumor, little or no additional information is gained with diagnostic CT after performance of PET/CT (2,19-21). As such, at least for lymphoma, the possibility that only PET/CT is necessary without any additional diagnostic CT was posited. This shift in clinical approach is intriguing and needs further delineation, especially for other tumor types.

Though such issues are still unsettled, we attempted to assess the actual diagnostic limitations in a group of oncologic patients undergoing PET/CT by comparing the findings with the subsequent diagnostic CT. Our goal was to determine limitations of $\mathrm{CT}^{\mathrm{p}}$ and to determine whether the current method of performance of PET/CT was optimal or could be improved. Finally, we aimed to assess whether all patients benefited from additional diagnostic full-body CT scans and, if not, which types of patients might need only limited, if any, additional scanning.

In our group of patients, the major cause for discrepant findings between PET/CT and diagnostic CT was the lack of intravenous contrast material. This most often led to undetected liver metastases or poor lymph node recognition and most often was apparent in patients who were likely to have liver metastases and undergo PET/CT - that is, patients with colorectal cancer. Some metastases could be seen on PET/CT if a wide window/level was applied to the CT image or if the metastases were large or metabolically active enough to be seen on PET. If further experience confirms that the intravenous contrast bolus can be given without interfering with CT attenuation mapping or tumor activity assessment, there should be no reason to perform duplicate CT scans and incur extra radiation and use extra time and resources unnecessarily. Work in progress suggests that PET/CT can be performed with intravenous contrast, despite earlier fears.

The second most common limitation of PET/CT compared with diagnostic CT in our group of patients occurred in the category of missed lung findings, either due to the occurrence of motion artifact from quiet respiration compared with breath-hold diagnostic CT or, surprisingly, due to the easily correctable reconstruction kernel used when displaying lung images. Although faster scanners with more detectors are being integrated, this will not overcome the motion artifacts from the quiet breathing technique used to match the PET acquisition.

The third most common reason for discrepant findings was the arm-down position used for all patients during PET/CT to allow comfort during prolonged PET and also used for the CT portion to avoid shifting anatomy and interference with anatomic coregistration. This resulted in a beam-hardening artifact most severe in the same horizontal plane in which the arms were resting. Development of faster more efficient collimator crystals could ideally allow for more rapid, arm-up PET as is done in routine diagnostic CT. Fortunately, the majority of our patients are now able to undergo arm-up positioning during the entire PET and CT acquisition.

Occasionally, a lack of oral contrast caused discrepant findings. However, it should be noted that $87 \%$ of cases were done without oral contrast, and the vast majority of discrepancies in these cases were unrelated to the lack of oral contrast. Thus, oral contrast seems to make little difference, probably because intestinal abnormalities are uncommon overall or because of the ease of identifying the intestines in all but the slimmest of patients.

The final category, accounting for only $4 \%$ of discrepancies, was the use of low mA.s for PET/CT. This was the reason that findings were thought to have been missed in only $4 \%$ of scans. This interesting, incidental observation is in keeping with suggestions in the literature that excessive doses of radiation are used routinely in CT scans and are unnecessary for diagnostic images (22-24).

A large number of discrepancies in this study were accounted for by perceptive, interpretive, or insignificant differences between the scans that did not represent true differences between the scans. Reader variability is common in interpretation of imaging studies, especially for such entities as abnormal lymph nodes at or around the $1-\mathrm{cm}$ size, as well as enlargement of organs such as the prostate, heart, and spleen, for which differing values are 
TABLE 3

Hepatic vs. Extrahepatic Findings (of 194 True Discrepancies)

\begin{tabular}{lccc}
\hline Malignancy & $\begin{array}{c}\text { Total no. of } \\
\text { discrepancies }\end{array}$ & $\begin{array}{c}\text { Hepatic } \\
(\%)\end{array}$ & $\begin{array}{c}\text { Extrahepatic } \\
(\%)\end{array}$ \\
\hline Colorectal & 72 & $29(40)$ & $43(60)$ \\
Lymphoma & 48 & $7(15)$ & $41(85)$ \\
Esophageal & 25 & $9(36)$ & $16(64)$ \\
Lung & 8 & $4(50)$ & $4(50)$ \\
Gastric & 8 & $2(25)$ & $6(75)$ \\
Cholangiocarcinoma & 7 & $5(71)$ & $2(29)$ \\
Neuroblastoma & 7 & $2(29)$ & $5(71)$ \\
Gallbladder & 5 & $4(80)$ & $1(20)$ \\
Pancreatic & 4 & $3(75)$ & $1(25)$
\end{tabular}

Data are expressed as number or number with percent in parentheses.

published. The seemingly large number of perceptive errors (most often multiple errors in only a few patients) we encountered consisted of 78 findings missed on retrospective review of $\mathrm{CT}^{\mathrm{p}}$ and 32 findings on the official report of $\mathrm{CT}^{\mathrm{d}}$. A sampling of these showed that despite the experience of the readers, these "nontrue" discrepancies were perhaps due to haste in reading cases or reader fatigue (e.g., a small subpleural lung nodule or a small inconspicuous liver lesion or heterogeneous body density) or differences in what radiologists consider important to mention in a report (e.g., a calcified subcarinal lymph node or cholelithiasis or a tiny kidney calculus without obstruction). We believe that these discrepancies can be found in any study involving inter- or intraobserver variability and do not reflect actual image-quality limitations such as we found in our other well-defined categories.

This study had some limitations. Our patients are from a tertiary cancer center that, on average, could have more advanced disease than the overall population of patients undergoing PET scans elsewhere. As such, additional metastases that were detected in this group on $\mathrm{CT}^{\mathrm{d}}$ but not on $\mathrm{CT}^{\mathrm{p}}$ may have made little difference to the overall stage or treatment. Second, in certain instances it was

TABLE 4

Hepatic vs. Extrahepatic Findings (of 60 Potentially Significant Discrepancies)

\begin{tabular}{lccc}
\hline Malignancy & $\begin{array}{c}\text { Total no. of } \\
\text { discrepancies }\end{array}$ & $\begin{array}{c}\text { Hepatic } \\
(\%)\end{array}$ & $\begin{array}{c}\text { Extrahepatic } \\
(\%)\end{array}$ \\
\hline Colorectal & 23 & $12(52)$ & $11(48)$ \\
Lymphoma & 16 & $3(19)$ & $13(81)$ \\
Esophageal & 7 & $3(43)$ & $4(57)$
\end{tabular}

Data are expressed as number or number with percent in parentheses. difficult to determine whether the lack of intravenous contrast or beam-hardening artifact (or both?) was the cause of the discrepant readings in some cases of obscured liver metastases. Possibly, an even greater number of discrepancies were due to the lack of intravenous contrast, only adding to the importance of using intravenous contrast. Finally, the reading of $\mathrm{CT}^{\mathrm{p}}$ in a study setting as opposed to the reading of $\mathrm{CT}^{\mathrm{d}}$ in a clinical setting may introduce a bias that results in a more accurate reading of $\mathrm{CT}^{\mathrm{p}}$ compared with $\mathrm{CT}^{\mathrm{d}}$. The likely effect of this would be a greater number of discrepancies-for example, missed liver lesions seen only after careful windowing performed here in the controlled readout session.

Although a larger study with cost/benefit analysis would be optimal, our study suggests that CT could be tailored during PET/CT in the following manner: (i) Patients with colorectal cancer and other epithelial primary malignancies will probably need full-body diagnostic scans because about one half of discrepancies occurred in sites outside of the liver, including small peritoneal implants and small lung nodules; (ii) For patients with lymphoma in whom nodal disease predominates and in which size estimates fail to predict disease on CT, our results parallel those in the literature, suggesting that the $\mathrm{CT}^{\mathrm{p}}$ scan that accompanies the PET scan may be adequate; (iii) For all patients if repeat $\mathrm{CT}^{\mathrm{d}}$ scanning is to be done, it could be limited to the chest and abdomen as little additional information seems to be gained from the pelvic portion of $\mathrm{CT}^{\mathrm{d}}$ scans; (iv) Although tailored strategies may be ideal, a more empiric and simple approach would be for all patients to undergo enhanced PET/CT as recent literature indicates that this is increasingly feasible.

\section{CONCLUSION}

In summary, we found that most discrepancies between the unenhanced, localizing, low-dose CT and the subsequent diagnostic CT were due to the lack of intravenous contrast, which obscured liver metastases and lymph node recognition. Easily altered technical factors could overcome the limitations of overall grainy images (low mA.s), focal beam-hardening (arm-down position), and poor-quality lung images (use of a non-edge-enhanced algorithm). Nonetheless, discrepant findings would have led to altered management in only 2 patients, suggesting that limited repeat imaging may be feasible and would have the added benefit of reducing radiation and use of valuable resources. Because workflow and economic concerns are part of our daily practice, those institutions that prefer to use unenhanced CT in PET/CT could do so confidently, particularly because past studies on lymphoma patients and our study suggest a limited need for follow-up scans using diagnostic CT. For those centers in which it is logistically feasible, conversion to the use of a normal $\mathrm{mA} \cdot \mathrm{s}$ dose, with oral and intravenous contrast-enhanced CT in PET/CT, could overcome all potential discrepancies and provide the additional 
TABLE 5

Thirteen Pelvic Discrepancies Among 194 True Discrepancies

\begin{tabular}{llcc}
\hline Malignancy & \multicolumn{1}{c}{ Discrepant finding } & Discrepant category & Significant \\
\hline Colorectal & Bladder diverticulum & $\mathrm{mA}$ & Seen on PET \\
Colorectal & Thickened bladder & IV & Yes \\
Colorectal & $<5$-mm sigmoid mesenteric nodule & Oral & Yes \\
Colorectal & Residual tumor & Oral & Yes \\
Colorectal & Rectal soft tissue & Oral & Yes \\
Colorectal & Sigmoid and transverse colon thickening & Oral & Yes \\
Colorectal & Enhancing mass in postoperative bed & IV & Yes \\
Lymphoma & Prominent ovarian vein & IV & Yes* \\
Lymphoma & Prevesical soft-tissue mass & IV & \\
Lymphoma & Pelvic ascites & Oral & IV \\
Lymphoma & Uterine lobulation & IV & Yes \\
Neuroblastoma & L common and R external iliac adenopathy & IV &
\end{tabular}

*Management change in this case = performance of cystoscopy to exclude bladder primary abnormality.

advantage of reducing patient radiation exposure, patient and institutional cost, and use of valuable and limited resources.

\section{REFERENCES}

1. Young SW, Turner RJ, Castellino RA. A strategy for contrast enhancement of malignant tumor using dynamic CT and intravascular pharmacokinetics. Radiology. 1980;137:137-147.

2. Rodriguez-Vigil B, Gomez-Leon N, Pinilla I, et al. PET/CT in lymphoma: prospective study of enhanced full-dose PET/CT versus unenhanced low-dose PET/CT. J Nucl Med. 2006;47:1643-1648.

3. Wahl RL, Quint LE. Cieslak Rd, Aisen AM, Koeppe RA, Meyer CR. Anatometabolic tumor imaging: fusion of FDG PET with CT or MRI to localize foci of increased activity. J Nucl Med. 1993;34:1190-1197.

4. Berthelsen AK, Holm S, Klausen TL, Andersen F, Hojgaard L. PET/CT with intravenous contrast can be used for PET attenuation correction in cancer patients. Eur J Nucl Med Mol Imaging. 2005;32:1167-1175.

5. Nakamoto Y, Chin BB, Kraitchman DL, Lawler AP, Marshall LT, Wahl RL. Effects of nonionic intravenous contrast agents at PET/CT imaging: phantom and canine studies. Radiology. 2003;227:817-824.

6. Antoch G, Freudenberg LS, Egelhof T, et al. Focal tracer uptake: a potential artifact in contrast-enhanced dual modality PET/CT scans. J Nucl Med. 2002;43: 1339-1342.

7. Yau YY, Chan WS, Tam YM, et al. Application of intravenous contrast in PET/ $\mathrm{CT}$ : does it really introduce significant attenuation correction errors? $\mathrm{J} \mathrm{Nucl}$ Med. 2005;46:283-291.

8. Beyer T, Antoch G, Bockisch A, Stattaus J. Optimized intravenous contrast administration for diagnostic whole body ${ }^{18}$ F-FDG PET/CT. J Nucl Med. 2005;46:429-435.

9. Antoch G, Freudenberg LS, Stattaus J, et al. Whole-body positron emission tomography-CT: optimized CT using oral and IV contrast materials. AJR. 2002; 179:1555-1560.

10. Mawlawi O, Erasmus JJ, Munden RF, et al. Quantifying the effect of IV contrast media on integrated PET/CT: clinical evaluation. AJR. 2006;186:308-319.
11. Wong TZ, Paulson EK, Nelson RC, Patz EF Jr, Coleman RE. Practical approach to diagnostic CT combined with PET. AJR. 2007;188:622-629.

12. Brechtel K, Klein M, Vogel M, et al. Optimized contrast-enhanced CT protocols for diagnostic whole-body ${ }^{18} \mathrm{~F}$-FDG PET/CT: technical aspects of single-phase versus multiphase CT imaging. J Nucl Med. 2006;47:470-476.

13. Dizendorf E, Hany TF, Buck A, Von Schulthess GK, Burger C. Cause and magnitude of error induced by oral CT contrast agent in CT-based attenuation correction of PET emission studies. J Nucl Med. 2003;44:732-738.

14. Dizendorf EV, Treyer V, Von Schultess GK, Hany TF. Application of oral contrast media in co registered positron emission tomography CT. AJR. 2002; 179:477-481.

15. Antoch G, Jentzen W, Freudenberg LS, et al. Effect of oral contrast agents on CT-based PET attenuation correction in dual-modality PET/CT imaging. Invest Radiol. 2003;38:784-789.

16. Antoch G, Kuehl H, Kanja J, et al. Dual-modality PET/CT scanning with negative oral contrast agent to avoid artifacts: introduction and evaluation. Radiology. 2004;230:879-885.

17. Huebner RH, Park KC, Sheperd JE, et al. A meta-analysis of the literature for whole-body FDG PET detection of recurrent colorectal cancer. J Nucl Med. 2000;41:1177-1189.

18. Ott K, Weber W, Siewert JR. The importance of PET in the diagnosis and response evaluation of the esophageal cancer. Dis Esophagus. 2006;19:433-442.

19. Steinert HC. PET/CT in lymphoma patients [in German]. Radiologe. 2004;44: 1060-1067.

20. Schaefer NG, Hany TF, Taverna C, et al. Non-Hodgkin lymphoma and Hodgkin disease: coregistered FDG PET and CT at staging and restaging-do we need contrast-enhanced CT? Radiology. 2004;232:823-829.

21. Choi H. Critical issues in response evaluation on computed tomography: lessons from the gastrointestinal stromal tumor model. Curr Oncol Rep. 2005;7: 307-311.

22. Yamada T, Ono S, Tsuboi M, Saito H, Sato A, Matsuhashi T, et al. Low-dose CT of the thorax in cancer follow-up. Eur J Radiol. 2004;51:169-174.

23. Zhu X, Yu J, Huang Z. Low dose chest CT: optimizing radiation protection for patients. AJR. 2004;183:809-816.

24. Kalra MK, Prasad S, Saini S, Blake MA, Varghese J, Halpern EK. Clinical comparison of standard-dose and 50\% reduced-dose abdominal CT: effect on image quality. AJR. 2002;179:1101-1106. 